\title{
BJS Open
}

Welcome to this first issue of BJS Open, an open access electronic journal closely allied to BJS. Open access publishing is becoming increasingly popular as researchers, funders, academic institutions and individual hospitals seek to have work made available to as wide an audience as possible, without the financial constraints that can limit accessibility to subscription journals. Although often referred to as 'author pays', an increasing number of research funders and academic institutions are providing these funds, but insisting on open access publication.

Traditional publication of scientific articles has largely involved a subscription model and, for many established journals, a regular print issue. The cost of the latter, postage and lack of immediacy are all problems that have been overcome in the era of electronic publication, but other factors can still limit subscription journals. The volume of material that can be published may be limited by the contract between publisher and the journal owner, particularly when a print journal is created. When a journal becomes popular with readers (BJS is a good example), space limitations make it inevitable that some high-quality work is turned down.

BJS Open reflects the views of its charitable owners, the British Journal of Surgery Society, and has been set up to complement the subscription journal, BJS. In terms of content, BJS Open is a true general surgical journal designed to have international appeal as global surgical problems become increasingly evident. A mixture of editorials, systematic reviews, randomized trials and other types of original articles will characterize the content over the first few years.
At the moment, acceptance for publication in BJS Open occurs by the transfer of submissions made to BJS. Submission to BJS ensures that all material undergoes a single peer review system. Where pressure of space limits the publication of high-quality material that is scientifically sound, authors receive an offer of transfer to BJS Open, with guaranteed publication as an open access article subject to making changes recommended by peer reviewers and the editor. For a limited period, the usual article publication charge payable by authors is being waived.

In time, commissioned reviews dealing with topical issues will appear and it is hoped that BJS Open will become associated with the publication of high-quality clinical trial protocols as well as the publication of results. It is also hoped that clinical guidelines, produced by collaborating societies, associations and academic partnerships, to encourage high standards of surgical care, may also find a home in the journal.

The value of social media to attract an audience of interested readers cannot be underestimated. While important for the wide dissemination of any scientific work, this approach is particularly relevant to open access publication. We hope that this becomes an important part of BJS Open activity. It will certainly help to shape the future of the Journal and we hope that you enjoy being involved. 